\title{
Utilization of Beta-Blockers and Diuretics in Treating Heart Failure Patients in Sultan Qaboos University Hospital
}

\author{
Tariq Dhiyab Al-Saadi, Al-Salt Al-Kharusi, and Ali Abdulrahman
}

\begin{abstract}
Background: Heart failure (HF) is the inability of the heart to pump blood to meet tissue requirement of oxygen and nutrition, due to abnormality in cardiac structure or function. The mortality from congestive heart failure remain increasing along with aging of the population. In Oman, the prevalence of $\mathrm{HF}$ is 5.17 per 1000 people and with male and older age group domination. Beta-blockers and diuretics are well known drugs that decrease mortality and morbidity. According to heart failure treating guidelines beta-blockers are the first line treatment for HF. Since HF has a high prevalence, this study aim was to determine the utilization of beta-blockers and diuretics in treating heart failure patients in Sultan Qaboos University Hospital (SQUH). Methods: A retrospective cross-sectional conducted in SQUH, in cardiology day care clinic in the period from 1st of June until 30th of August. This study included all Omani patients with 120 out of 778 patients were included who were above 18 years old, diagnosed with $\mathrm{HF}$, receiving at least one $\mathrm{HF}$ medication from SQUH pharmacy, and did not have any missing data. Hospital electronic medical record was used to obtain patients data and parameters. Results: Out of 120 patients, $54.2 \%$ were males and $45.8 \%$ were females, with mean age equal to $64 \pm 13$ years and mean EF equal to $37 \pm 14.5 \%$. Diuretics were taken by $95 \%$ of the patients, where beta-blockers were taken by $81.6 \%$. Prescription of beta-blockers was significantly decreased by increase in the age of the patients $(p=0.024)$, while diuretics do not show any significant with change in the age. Both betablockers and diuretics do not show any significant increase or decrease in prescription with change in the EF. Bisoprolol was not affected by increase in age or EF. The prescription of carvedilol was significantly affected with increase the age $(p=$ 0.006), however it was not affected by increase the EF. The prescription of spironolactone was significantly affected by increase the age $(P=0.001)$ and by increase the EF $(P=0.001)$. Conclusion: Carvedilol and furosemide were the most prescribed drugs in SQUH for treating HF patients. All the mean daily doses of the drugs mentioned in this study followed the newer 2016 European Society of Cardiology Guidelines for the diagnosis and treatment of acute and chronic $\mathrm{HF}$.
\end{abstract}

Index Terms-Heart Failure, Ejection Fraction, Hypertension, SQUH, Diuretic.

\section{BACKGROUND}

\section{A. Heart failure:}

Heart failure (HF) is the inability of the heart to pump blood to meet tissue requirement of oxygen and nutrition,

Published on April 21, 2020.

Tariq Dhiyab Al-Saadi, McGill University, Canada. (corresponding email: t.dhiyab@hotmail.com )

Al-Salt Al-Kharusi, Sultan Qaboos University, Oman.

(email: Saltsaid9@gmail.com)

Ali Abdulrahman, Sultan Qaboos University, Oman. due to abnormality in cardiac structure or function (Dickstein et al., 2010). Clinically, it can be defined as a syndrome characterized by typical symptoms such as fatigue, swelling, and breathless, and typical signs such as raised jugular venous pressure, displaced apex beat. The result of these symptoms and signs is abnormality in cardiac structure and function (McMurray et al., 2012). HF is classified into 4 classes according to the New York Heart Association and patient symptoms used in the classification. It could be also classified according to cardiac cycle into systolic and diastolic heart failure (Nicholson, 2014). Hypertension and ischemic heart disease (IDH) are the most well-known predisposing factors for HF (Al-Shamiri, 2013). However, it could be caused by idiopathic cardiomyopathy, arrhythmias, coronary artery disease, myocarditis, valve disorders, alcohol-induced cardiomyopathy, amyloidosis, genetic cardiomyopathies, and metabolic disorders. Obesity and diabetes mellitus type I and II are also considered as contributing factors (Nicholson, 2014) ( Al-Shamiri, 2013).

Over the last decade, there was a noticeable decrease in mortality caused by cardiovascular diseases, with about $27 \%$ decrease in mortality caused by myocardial infraction. Despite that, mortality from congestive heart failure remain increasing along with aging of the population (Levy \& Levy, 2002). In developed countries, the prevalence of HF tend to be low (1\%-2\%) in adult's populations with incidence of 5-10 per 1000 annually and increase to (>10\%) with older populations (Mosterd \& Hoes, 2007). This can emphasize that $\mathrm{HF}$ is an age-related disease as it well known cause for mortality and morbidity especially in people aged above 65 years (Rich, 1997). A hospital-based retrospective study conducted in Oman, showed that the prevalence of HF is 5.17 per 1000 people and with male and older age group domination (Agarwal et al., 2001).

\section{B. Pharmacology treatment}

There is a wide variety of drugs that are used in the management of HF, which aim to reduce the mortality and morbidity and improve patient's physical and psychosocial status and ultimately improve patient's quality of life (Doba et al, 1999). In heart failure, there is activation of the sympathetic nervous system and renin-angiotensin system (RAS), so inhibition of these has become important therapeutically (Hunt et al., 2005). The pharmacological treatment includes these groups of drugs: beta-blockers, angiotensin receptor blockers (ARBs), diuretics, angiotensin-converting enzymes inhibitors (ACEI) (AlShamiri, 2013). Inotropic agents are also found to be effective in treating heart failure patients (Levy \& Levy, 2002). 


\section{Beta blockers}

Beta blockers, also known as beta-adrenergic antagonists, showed improvement in patient's quality of life and functional status survival (Packer et al., 1996). A metaanalysis study showed a significant reduction in mortality, sudden death, and cardiac death by $29.6 \%, 49.4 \%$, and $29.8 \%$ respectively while using beta-blockers in HF patients (He et al., 2012). Beta-blockers inhibit beta receptors which are found throughout the body, especially in the heart which has beta 1 receptors. Beta blockers had got three generations, first generation of beta blockers was nonselective which used to block beta 1 and 2 receptors, second generation used to be selective and block beta 1 receptor only, while the third generation has both selectivity and nonselectivity ability (Westfall \& Westfall, 2011). Many beta blockers clinical trials showed that using beat blocker will improve the function of left ventricle and decrease heart failure patients' symptoms (Bruns LA \& Carter CE, 2002 ,6). Upregulation the beta receptors and prevent cardiac remodeling can be achieved by inhibiting of beta receptor (Bruns \& Carter, 2002). Carvedilol is a non-selective beta blocker with alpha 1 receptor blocking characteristic which is beneficial for heart failure patients as it decreases heart rate and peripheral vessel resistance (Westfall \& Westfall, 2011). Carvedilol is given orally as it is absorbed from the stomach epithelium, and metabolized by the liver through cytochrome p450 isoenzyme CYP2D6 (Shihmanter R et al, 2014). The half-life of carvedilol was found to be increasing with increase in the age (Laer S, et al, 2002), however the bio-availability increase with those patients with heart failure. Bisoprolol is a selective beta 1 receptor blocker, and it is beneficial for HF patients as it decreases the heart rate (Trobec et al, 2016). Bisoprolol is metabolized by the liver and excreted through the kidney. Some studies showed that pharmacokinetics of this drug is affected by body composition (Trobec et al, 2016).

\section{Diuretics}

Diuretics are effective in the management of HF. This goal is achieved by using the most appropriate class of diuretics or a combination of them, convenient dosage and monitoring their side effects (A National Clinical Guideline,2016). Diuretics are divided into three classes loop diuretics, thiazides, and potassium sparing. Loopdiuretics (e.g. furosemide) are preferred over other diuretics and are commonly used as they maintain their effect in low renal function, (Dickstein et al., 2010). Loop-diuretics act by inhibiting $\mathrm{Na}^{+} / \mathrm{K}^{+} / 2 \mathrm{Cl}^{-}$co-transporter in ascending limb of the loop of Henle and increase sodium and water excretion (Rang et al., 2011). Thiazides (e.g. bendroflumethiazide) and thiazides like diuretics (e.g. metalozone) (Dickstein et al., 2010), act at the distal convoluted tubule to inhibit reabsorption of sodium chloride and increase sodium and water excretion (Rang et al., 2011). Potassium-sparing diuretics (e.g. spironolactone, amiloride) are considered as weak diuretics, but they are used in combination with other diuretics for hypokalemia correction (Dickstein et al., 2010).

\section{METHODS}

This retrospective cross-sectional study has been conducted in Sultan Qaboos University Hospital (SQUH), from $1^{\text {st }}$ of June until $30^{\text {th }}$ of August 2016. This study targeting those patients who are diagnosed with HF and taking pharmacological treatment from SQUH pharmacy. Ethical approval was obtained from the Sultan Qaboos University Ethical Review Committee. Furthermore, the access authorization to the hospital electronic medical records (TrackCare System) was provided by the Hospital Information System (HIS). From cardiology day clinic in SQUH, 778 patients were collected, and by using the hospital electronic medical record system which has been used in the hospital since 2006, we were able to exclude all patients who were non-Omani or with age less than 18 as well as those who had missing or unclear data. Out of 778 patients, 120 patients were only included in this study.

The collected data has been classified into three main sections. The first section contains all the information that are related to the patient which include gender, age in years, alcoholic and smoking status, and the possible family history. The second section contain the patient heart failure condition included duration of the disease in years, value of the ejection fraction in percentage, and NYHA classification of heart failure from I to IV. In addition, the laboratory tests were also collected include: estimated glomerular filtration rate (eGFR), sodium $(\mathrm{Na}+)$ in $\mathrm{mmol} / \mathrm{liter}$ and potassium $(\mathrm{k}+)$ in $\mathrm{mmol} /$ liter balance to determine the kidney function and the possible effect on the drugs, also serum brain natriuretic peptide (BNP) in mmol/liter which used as a marker for myocardial muscle damage. The last section, which is the main section in this study contains the patient pharmacological treatment.

All the gathered data has been processed and analyzed by Statistical Package for the Social Sciences (SPSS) program. Different Statistical tests were used include chi-square test which was used to test for the significant differences and one-way analysis of variance (ANOVA) test which was used to compare the means of the drug groups with different age and EF groups. Significance level of 0.05 was estimated for the obtained results. In addition, statistical parameters which include mean, standard deviation (SD) and standard error (SE) were gathered using this program. while Statistical Package for the Social Sciences (SPSS) program was used for Statistical tests, pie and bars charts were created by Microsoft Office Excel program.

Age parameter was divided into four main groups according to the 2016 European Society of Cardiology (ESC) Guidelines for the diagnosis and treatment of acute and chronic heart failure (ESC Guidelines, 2016). The groups were (Age 1) <60 years, (Age 2) 60-70 years, (Age 3) $70-80$ years, and (Age 4) $>80$ years. Ejection Fraction also divided into three groups according to 2016 European Society of Cardiology (ESC) Guidelines for the diagnosis and treatment of acute and chronic heart failure (ESC Guidelines, 2016) and these groups are heart failure with reduced ejection fraction (HFrEF) with Ejection Fraction less than $40 \%$, heart failure with mid-range ejection fraction (HFmrEF) with Ejection Fraction from 40 to $49 \%$ and heart failure with preserved ejection fraction (HFpEF) with Ejection Fraction more than 50\%. 


\section{RESUltS}

\section{A. Demographic data}

TABLE I: DEMOGRAPHIC DATA

\begin{tabular}{|c|c|c|c|c|}
\hline \multicolumn{2}{|c|}{ Parameter (Unit) } & N (\%) & $\begin{array}{l}\text { Mean } \pm \text { Standard } \\
\text { Deviation }\end{array}$ & Range \\
\hline \multirow[b]{2}{*}{ Nationality } & Omani & $120(100 \%)$ & & \\
\hline & $\begin{array}{l}\text { Non- } \\
\text { Omani }\end{array}$ & $0(0 \%)$ & & \\
\hline Age (years) & & $120(100 \%)$ & $64 \pm 13$ & $\begin{array}{l}25- \\
92\end{array}$ \\
\hline \multirow{4}{*}{ Age groups } & Age 1 & $40(33.3 \%)$ & $50 \pm 9$ & $\begin{array}{l}25- \\
49\end{array}$ \\
\hline & Age 2 & $41(34.2 \%)$ & $64 \pm 3$ & $\begin{array}{l}60- \\
70\end{array}$ \\
\hline & Age 3 & $28(23.3 \%)$ & $75 \pm 3$ & $\begin{array}{l}71- \\
79 \\
\end{array}$ \\
\hline & Age 4 & $11(9.2 \%)$ & $85 \pm 5$ & $\begin{array}{l}80- \\
92 \\
\end{array}$ \\
\hline \multirow{2}{*}{ Gender } & Male & $65(54.2 \%)$ & & \\
\hline & Female & $55(45.8 \%)$ & & \\
\hline \multicolumn{2}{|l|}{$\mathrm{EF}(\%)$} & $119(99.2 \%)$ & $37 \pm 14.5$ & $\begin{array}{l}10- \\
78\end{array}$ \\
\hline \multirow{3}{*}{ EF groups } & HFrEF & $79(66.4 \%)$ & $28.8 \pm 6.9$ & $\begin{array}{l}10- \\
39\end{array}$ \\
\hline & HFmrEF & $19(16 \%)$ & $43.7 \pm 3.3$ & $\begin{array}{l}40- \\
49\end{array}$ \\
\hline & HFpEF & $21(17.6 \%)$ & $61.7 \pm 9.6$ & $\begin{array}{l}50- \\
78 \\
\end{array}$ \\
\hline \multirow[b]{2}{*}{ Smoking } & Smoker & $17(14.2 \%)$ & & \\
\hline & $\begin{array}{l}\text { Non- } \\
\text { Smoker }\end{array}$ & $103(85.8 \%)$ & & \\
\hline \multirow[b]{2}{*}{$\begin{array}{l}\text { Alcohol } \\
\text { Consumption }\end{array}$} & Alcoholic & $6(5 \%)$ & & \\
\hline & $\begin{array}{l}\text { Non- } \\
\text { Alcoholic }\end{array}$ & $114(95 \%)$ & & \\
\hline \multicolumn{2}{|c|}{ NYHA Classification } & $14(11.7 \%)$ & & \\
\hline \multirow{4}{*}{$\begin{array}{l}\text { NYHA } \\
\text { Classes }\end{array}$} & Class I & $2(14.3 \%$ & & \\
\hline & Class II & $7(50 \%)$ & & \\
\hline & Class III & $4(28.6 \%)$ & & \\
\hline & Class VI & $1(7.1 \%)$ & & \\
\hline \multicolumn{2}{|c|}{$\begin{array}{l}\text { Duration of heart failure } \\
\text { (Years) }\end{array}$} & $120(100 \%)$ & $2.24 \pm 1.67$ & $1-10$ \\
\hline \multicolumn{2}{|c|}{$\begin{array}{l}\text { Estimated Glomerular } \\
\text { Filtration Rate (mL/min) }\end{array}$} & $118(98.3 \%)$ & $65.36 \pm 20.79$ & $\begin{array}{l}23- \\
>90\end{array}$ \\
\hline \multicolumn{2}{|c|}{ Serum Potassium (mmol/L) } & $120(100 \%)$ & $4.43 \pm 0.46$ & $\begin{array}{l}3.3- \\
6.2 \\
\end{array}$ \\
\hline \multicolumn{2}{|c|}{ Serum Sodium $(\mathrm{mmol} / \mathrm{L})$} & $120(100 \%)$ & $137.47 \pm 3.90$ & $\begin{array}{l}124- \\
145\end{array}$ \\
\hline \multicolumn{2}{|c|}{$\begin{array}{l}\text { Serum Brain Natriuretic } \\
\text { Peptide }(\mathrm{mmol} / \mathrm{L})\end{array}$} & $52(43.3 \%)$ & $2482.46 \pm 3448.84$ & $\begin{array}{l}37- \\
18571 \\
\end{array}$ \\
\hline \multicolumn{5}{|c|}{$\begin{array}{l}\text { (HFrEF) heart failure with reduced ejection fraction }(<40 \%) \text {, (HFmrEF) } \\
\text { heart failure with mid-range ejection fraction }(40-49 \%) \text {, (HFpEF) heart } \\
\text { failure with preserved ejection fraction }(>49 \%) \text {. (Age } 1)<60 \text { years, (Age } 2) \\
60-70 \text { years, (Age } 3) 70-80 \text { years, (Age } 4)>80 \text { years. (NYHA) New York } \\
\text { Heart Association }\end{array}$} \\
\hline
\end{tabular}

Table I shows the demographic data of the sample that has been studied. The study includes 120 Omani patients and 0 non-Omani, with dominance of the male $(54.2 \%)$. The duration of heart failure ranged from 1 year to 10 years. The age of the patients ranges from 25 to 92 with mean equal to 64 and standard deviation equal to 13. Categorization of the age showed, that the majority of the patients were in the first two groups and the minority of them in the last two. Ejection fraction of 119 patients showed that the it ranges from $10 \%$ to $78 \%$. Most of the patients had heart failure with reduced ejection fraction $(<40 \%)$. Out of 120 patients, 17 patients were smokers and 6 were alcoholic. New York Heart Association classification (Attached in the appendix) of 14 patients showed that 7 patients were in the class II.

\section{B. Heart failure}

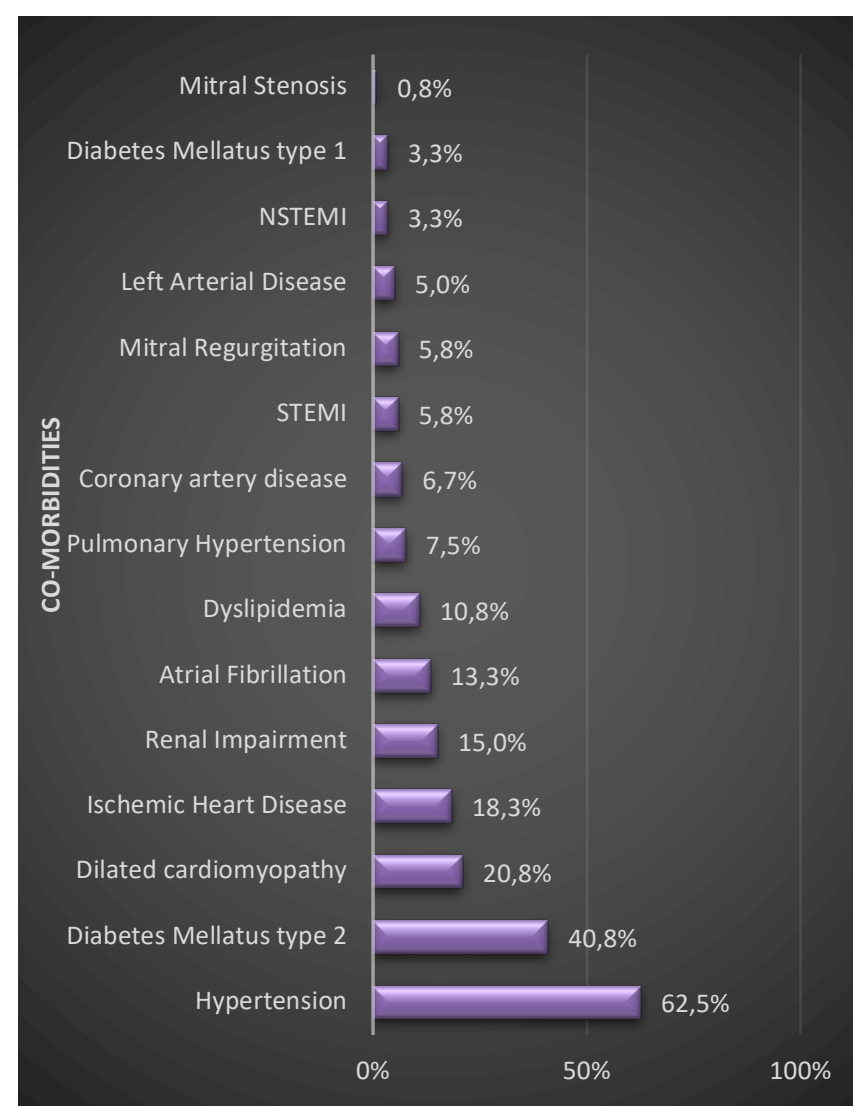

Fig. 1. Percentage (\%) of different co-morbidities among heart failure patients in SQUH.

(STEMI) elevated-ST myocardial infarction. (NSTEMI) not-elevated-ST myocardial infarction.

This figure shows the diseases that are related to the heart and seen among the heart failure patients who were included in the study. Hypertension dominated $(62.5 \%)$ and diabetes mellitus type 2 in the second $(40.8 \%)$. Dilated cardiac myopathy follows the diabetes type $2(20.8 \%)$, ischemic heart disease $(18.3 \%)$, renal impairment $(15 \%)$, atrial fibrillation $(13.3 \%)$, dyslipidemia $(10.8 \%)$, pulmonary hypertension $(7.5 \%)$, coronary heart disease $(6.7 \%)$, STEMI $(5.8 \%)$, mitral regurgitation $(5.8 \%)$, left arterial disease (5.0\%), NSTEMI (3.3\%), diabetes mellitus type 1 (3.3\%), and the least disease seen was mitral stenosis $(0.8 \%)$.

\section{Beta-blockers and diuretics}

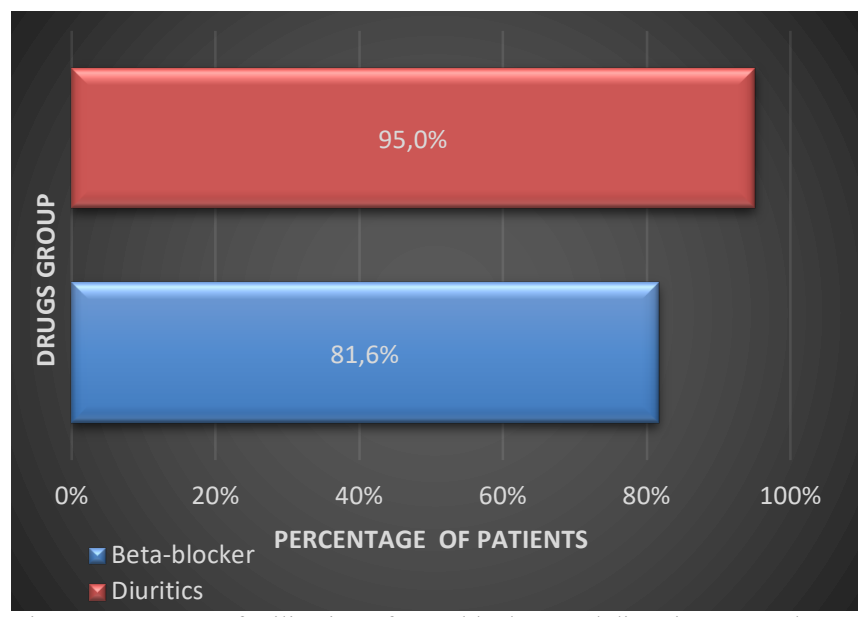

Fig. 2. Percentage of utilization of Beta-blockers and diuretics among heart failure patients in SQUH. 
Fig. 2 illustrates the utilization of beta blockers and diuretics among the heart failure patients in SQUH. From the figure $95 \%$ (114 out of 120 patients) were on diuretics medications, while beta-blockers were utilized by $81.6 \%$ (98 out of 120 patients).

\section{Beta-blockers}

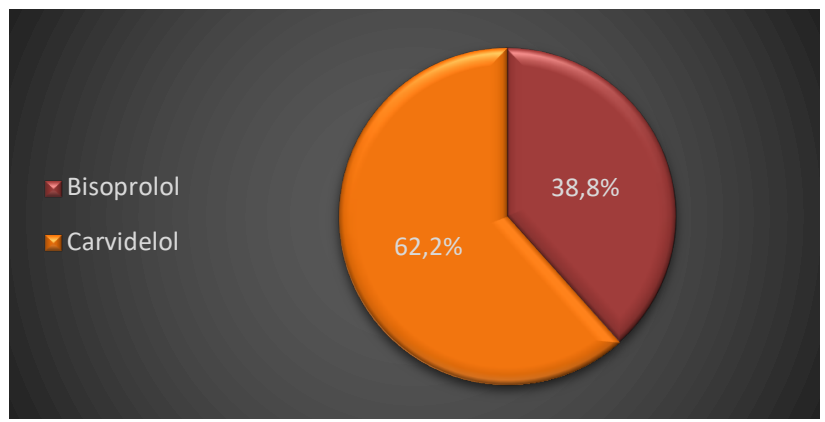

Fig. 3. Percentage of patients who were on beta-blockers medications among heart failure patients in SQUH.

Fig. 3 shows that out of 98 patients who were on betablockers $37.8 \%$ (38 patient) received bisoprolol and $62.2 \%$ (61 patients) were on carvedilol.

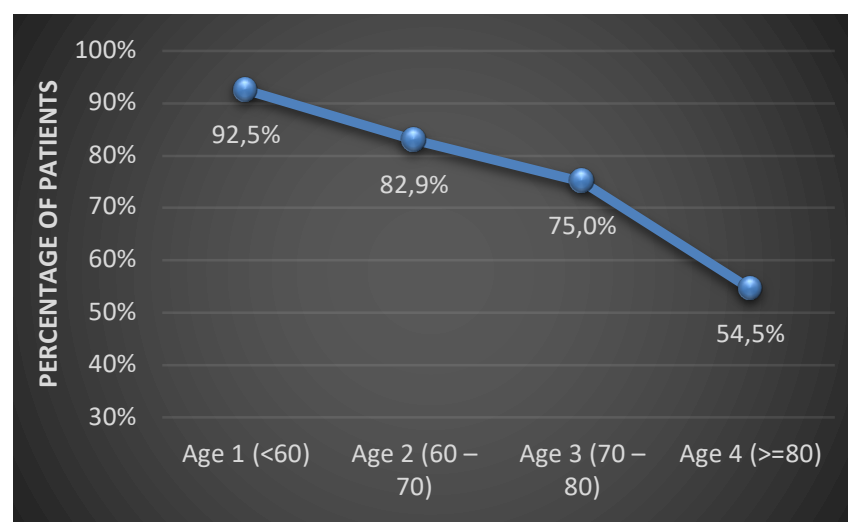

Fig. 4. Percentage of utilization of beta-blockers among heart failure patients in SQUH according to different age groups.

(Age 1) $<60$ years, (Age 2) 60-70 years, (Age 3) 70-80 years, (Age 4) $>80$ years. (NYHA) New York Heart Association.

Fig. 4 shows that $92.5 \%$ of the patients who were in the first age group received beta-blockers however this percentage decreased to $82.9 \%$ in the second group and to $75 \%$ in the third age group. In the fourth age group the utilization decreased to $54.5 \%$. This decrease in utilization of beta-blockers in the different age groups was significant $(P$ value $=0.024)$

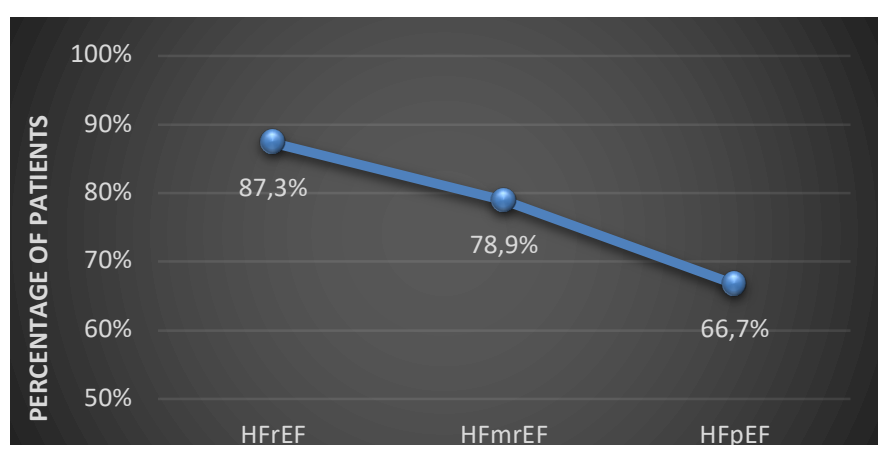

Fig. 5. Percentage of utilization of beta-blockers among heart failure patients in SQUH according to different ejection fraction groups. (HFrEF) heart failure with reduced ejection fraction $(<40 \%)$, (HFmrEF) heart failure with mid-range ejection fraction (40-49\%), (HFpEF) heart failure with preserved ejection fraction $(>49 \%)$
Fig. 5 shows that the patients with HFrEF have the highest utilization of beta-blockers $(87.3 \%)$ and this percentage decreased to $78.9 \%$ in patients with HFmrEF. This percentage decreased furthermore to reach $66.7 \%$ in the patients HFpEF. There was no significant difference in the utilization of beta blockers in different ejection fraction groups $(P$ value $=0.917)$.

\section{E. Bisoprolol}

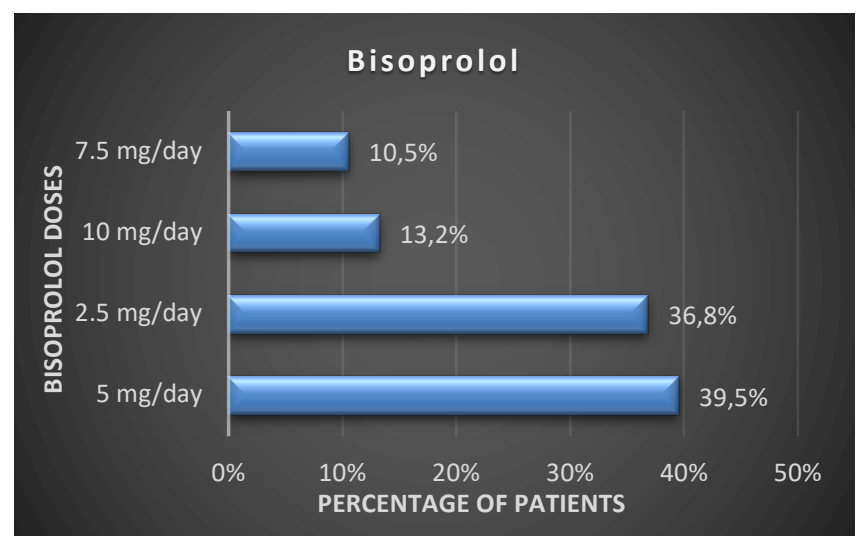

Fig. 6. Percentage of bisoprolol daily doses prescribed for heart failure patients in SQUH.

Fig. 6 shows the daily doses of bisoprolol given to heart failure patients. There were four doses $2.5,5,7,5$ and 10 $\mathrm{mg}$ /day prescribed to heart failure patients. $39.5 \%$ (15 out of 38 patients) were on $5 \mathrm{mg} /$ day, $36.8 \%$ (14 out of 38 patients) were on $2.5 \mathrm{mg} /$ day, $13.2 \%$ (5 out of 38 patients) were on 10 $\mathrm{mg} /$ day and $10.50 \%$ (4 out of 38 patients) were on 7.5 gm/day.

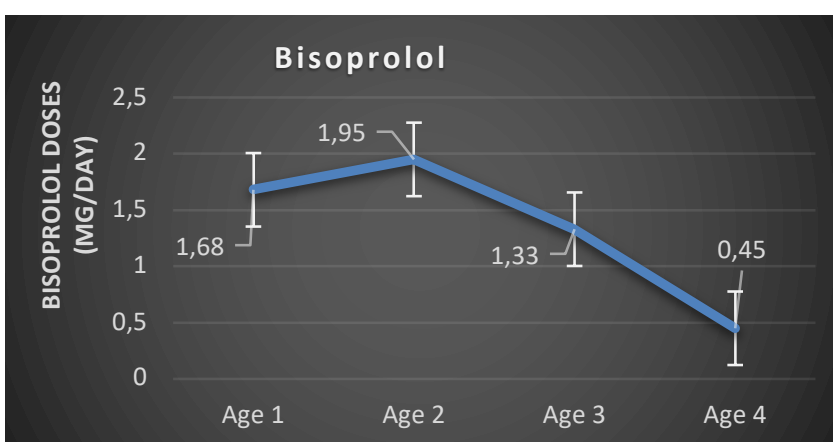

Fig. 7. Mean \pm SE of bisoprolol daily doses with different age groups. (Age 1) $<60$ years, (Age 2) 60-70 years, (Age 3) 70-80 years, (Age 4) $>80$ years. (NYHA) New York Heart Association.

Fig. 7 illustrates the change in the mean of daily doses of bisoprolol prescribed to heart failure patients according to different age groups. The mean of the daily doses for the first age group was $1.68 \mathrm{mg} /$ day and increased to 1.95 $\mathrm{mg} /$ day in the second age group. However, the mean daily doses of the third and fourth age groups decreased dramatically to reach $1.33 \mathrm{mg} /$ day and $0.45 \mathrm{mg} /$ day respectively. There was no significant difference in the bisoprolol means daily doses in the different age groups $(P$ value $=0.41)$ 


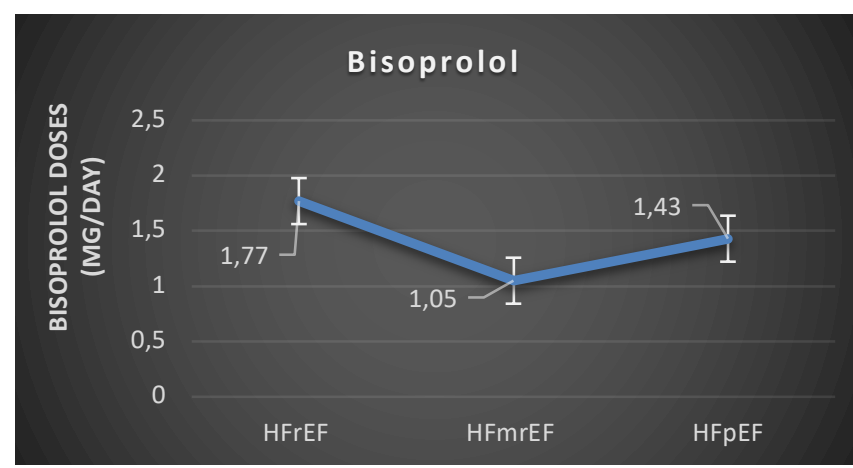

Fig. 8. Mean \pm SE of bisoprolol daily doses with different ejection fraction groups

(HFrEF) heart failure with reduced ejection fraction $(<40 \%)$, (HFmrEF) heart failure with mid-range ejection fraction (40-49\%), (HFpEF) heart failure with preserved ejection fraction $(>49 \%)$.

Fig. 8 illustrates the change in the mean of daily doses of bisoprolol prescribed to heart patients according to the different ejection fraction groups. The mean of the daily doses for HFrEF group was $1.77 \mathrm{mg} /$ day. However, the mean decrease to $1.05 \mathrm{mg} /$ day in the HFmrEF group. HFpEF showed slight increase in the mean daily doses of bisoprolol to reach to $1.43 \mathrm{mg} /$ day. The change in the means of daily doses of bisoprolol between the different ejection fraction groups is not significant $(P$ value $=0.565)$.

\section{F. Carvedilol}

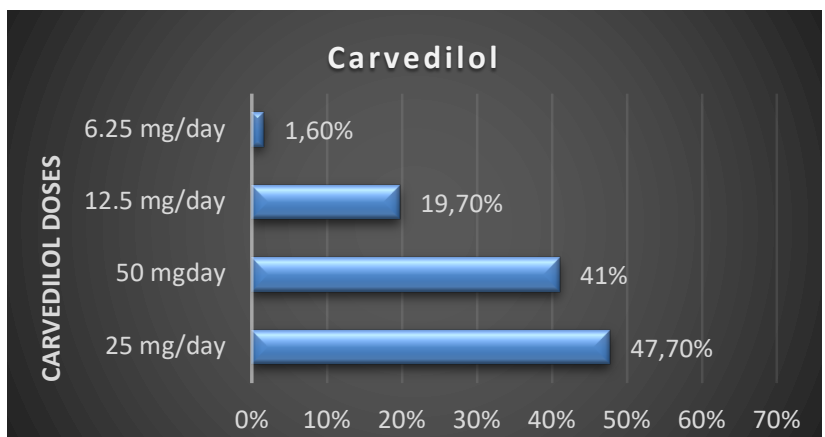

Fig. 9. Percentage of carvedilol daily doses prescribed for heart failure patients in SQUH

Fig. 9 shows the daily doses of carvedilol given to heart failure patients. There were four doses $6.25,12.5,25$ and 50 $\mathrm{mg}$ /day prescribed to heart failure patients. $47.7 \%$ (29 out of 61 patients) were on $25 \mathrm{mg} /$ day, $41 \%$ ( 25 out of 61 patients) were on $50 \mathrm{mg} /$ day, $19.7 \%$ (12 out of 61 patients) were on $12.5 \mathrm{mg}$ /day and $1.6 \%$ (1 out of 61 patients) were on 6.25 $\mathrm{gm} / \mathrm{day}$.

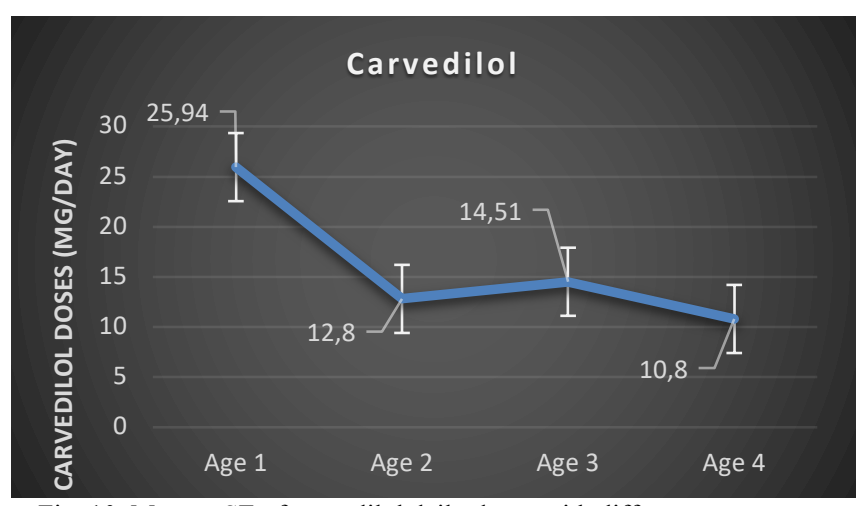

Fig. 10. Mean \pm SE of carvedilol daily doses with different age groups (Age 1) <60 years, (Age 2) 60-70 years, (Age 3) 70-80 years, (Age 4) >80 years. (NYHA) New York Heart Association
Fig. 10 illustrates the change in the mean of daily doses of carvedilol prescribed to heart failure patients according to the different age groups. The mean of daily doses for the first age group was $25.94 \mathrm{mg} /$ day and it decreased to 12.8 $\mathrm{mg}$ /day in the second age group, however the mean daily doses of the third group increased slightly to reach 14.51 $\mathrm{mg} /$ day and again decreased in the fourth age groups to reach $10.8 \mathrm{mg} /$ day. There was significant difference in the means between the age groups $(P$ value $=0.006)$.

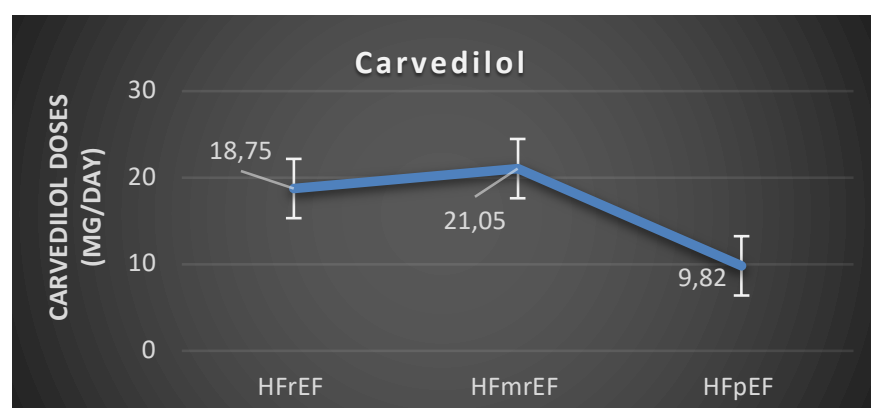

Fig. 11. Mean \pm SE of carvedilol daily doses with different ejection fraction groups.

(HFrEF) heart failure with reduced ejection fraction ( $<40 \%)$, (HFmrEF) heart failure with mid-range ejection fraction $(40-49 \%)$, (HFpEF) heart failure with preserved ejection fraction $(>49 \%)$.

Fig. 11 shows the change in the mean of the daily doses of carvedilol prescribed to heart patients according to different ejection fraction groups. The mean doses for HFrEF group was equal to $18.75 \mathrm{mg} /$ day. However, the mean daily doses increased slightly to $21.05 \mathrm{mg}$ /day in the HFmrEF group. In HFpEF group, there was a dramatic decrease in the mean of the daily doses and it reached to $9.82 \mathrm{mg} /$ day. There was no significant difference in means between different ejection fraction groups $(P$ value $=0.117)$.

\section{G. Diuretics}

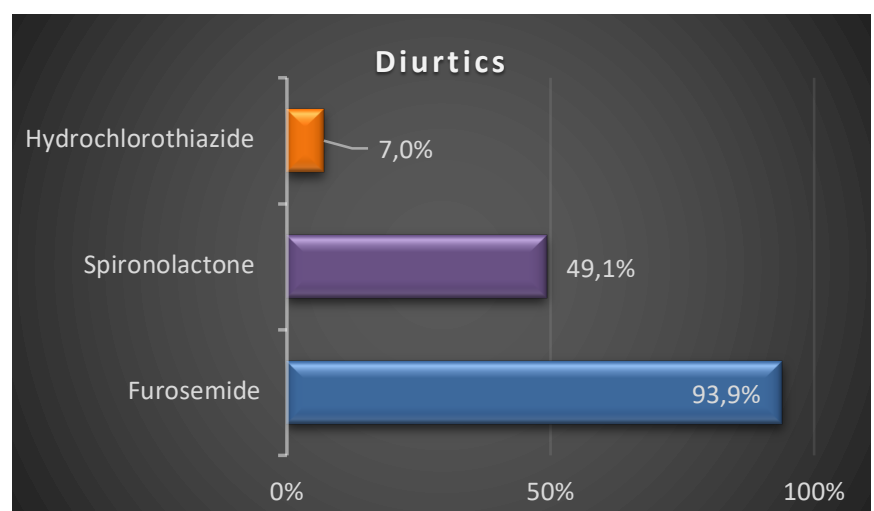

Fig. 12. Percentage of patients who were on diuretics medications among heart failure patients in SQUH.

Fig. 12 shows that out of 114 patients who were on diuretics $93.9 \%$ (107 patient) took Furosemide, 49.1\% (56 patients) were on spironolactone and $7.0 \%$ (8 patients) were on hydrochlorothiazide. 


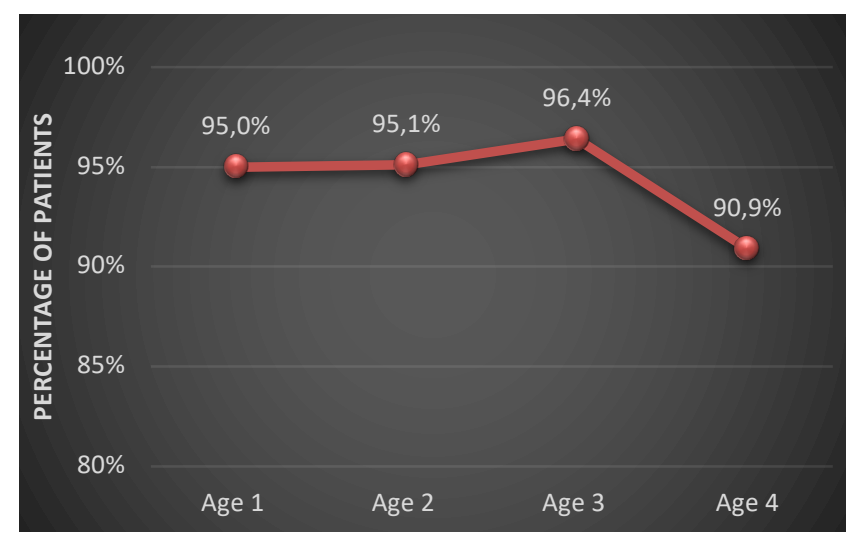

Fig. 13. Percentage of utilization of diuretics among heart failure patients in SQUH according to different age groups.

(Age 1) $<60$ years, (Age 2) 60-70 years, (Age 3) 70-80 years, (Age 4) $>80$ years. (NYHA) New York Heart Association.

Fig. 13 shows that $95 \%$ of the people who were in the first age group were on diuretics and $95.1 \%$ in the second age group, however the percentage of utilization increased slightly in the third age group and It reached $96.4 \%$. On the fourth group the percentage showed a slight decrease and it reached $90.9 \%$. There was no significant difference in the utilization of diuretics between different age groups. ( $P$ value $=0.917$ ).

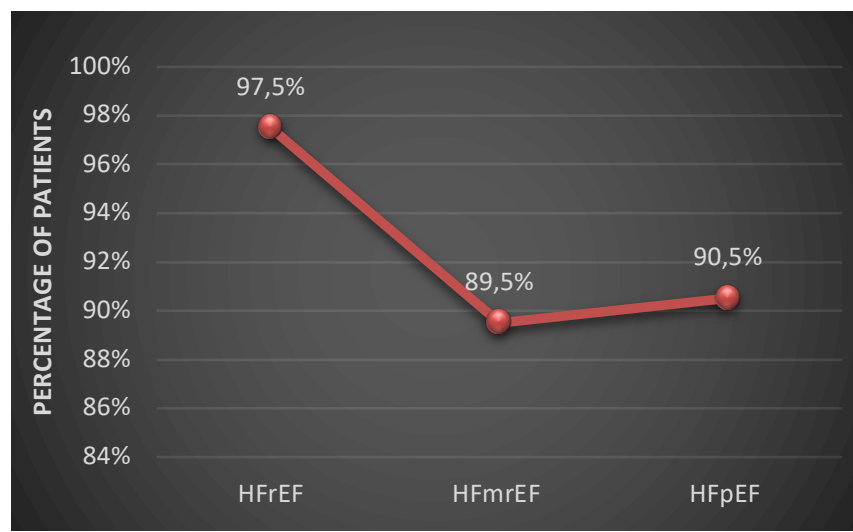

Fig. 14. Percentage of utilization of diuretics among heart failure patients in SQUH according to different ejection fraction groups.

(HFrEF) heart failure with reduced ejection fraction $(<40 \%)$, (HFmrEF) heart failure with mid-range ejection fraction $(40-49 \%)$, (HFpEF) heart failure with preserved ejection fraction $(>49 \%)$.

Fig. 14 shows that patients with HFrEF have the highest percentage of utilization of diuretics $(97.5 \%)$, this percentage decrease to $89.5 \%$ in patients with HFmrEF. However, Slight increase was seen in the percentage of utilization of diuretics in the patients with $\mathrm{HFpEF}$ and it reached to $90.5 \%$. The decrease in the utilization of diuretic in relation to ejection fraction groups showed no significant $(P$ value $=0.211)$.

\section{H. Furosemide}

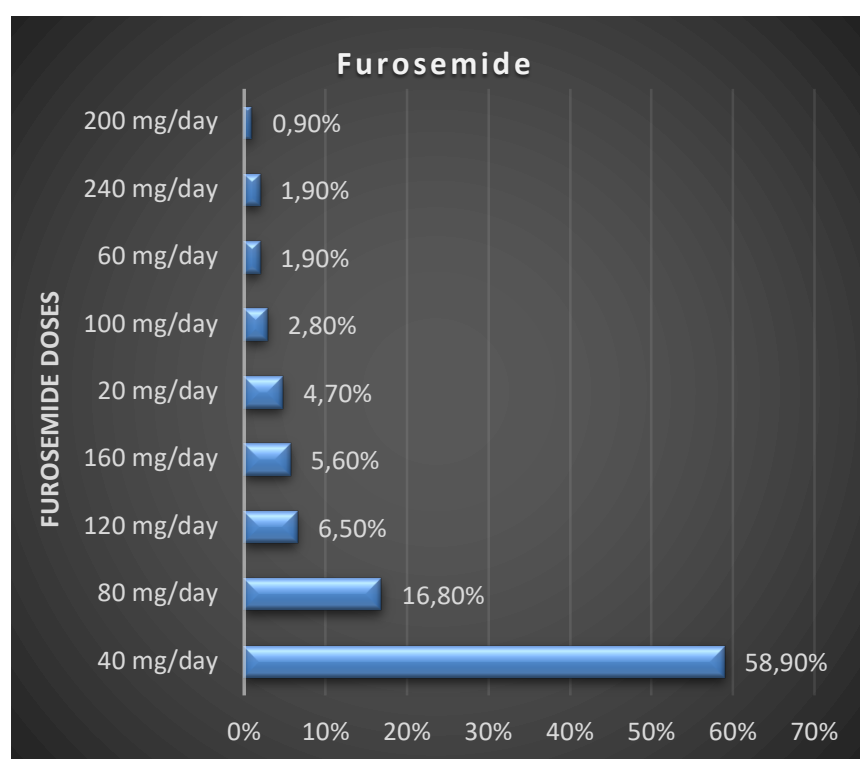

Fig. 15. Percentage of furosemide daily doses prescribed for heart failure patients in SQUH

Fig. 15 shows the daily doses of furosemide given to heart failure patients. There were nine doses $20,40,60,80$, $100,120,160,200$ and $240 \mathrm{mg} /$ day prescribed to heart failure patients. $58.9 \%$ (63 out of 107 patients) were on 40 $\mathrm{mg} /$ day, $16.8 \%$ (18 out of 107 patients) were on $80 \mathrm{mg} /$ day, $6.5 \%$ ( 7 out of 107 patients) were on $120 \mathrm{mg} /$ day, $5.6 \%$ (6 out of 107 patients) were on $160 \mathrm{gm} /$ day, 4.7\% (5 out of 107 patients) were on $20 \mathrm{gm} / \mathrm{day}, 2.8 \%$ ( 3 out of 107 patients) were on $100 \mathrm{gm} /$ day, $1.9 \%$ ( 2 out of 107 patients) were on $60 \mathrm{gm} /$ day, $1.9 \%$ (2 out of 107 patients) were on 240 $\mathrm{gm} /$ day and $0.9 \%$ ( 1 out of 107 patients) were on 200 gm/day.

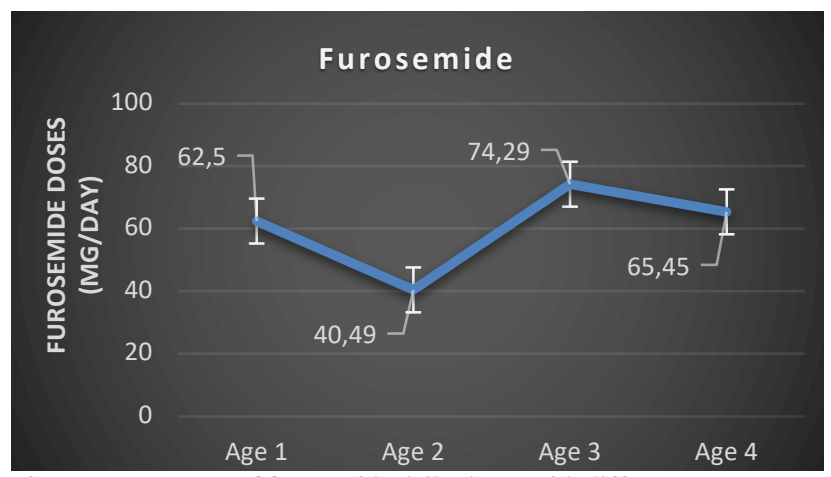

Fig. 16. Mean \pm SE of furosemide daily doses with different age groups. (Age 1) $<60$ years, (Age 2) 60-70 years, (Age 3) 70-80 years, (Age 4) $>80$ years. (NYHA) New York Heart Association.

Fig. 16 shows the change in the mean of daily doses of furosemide prescribed to heart failure patients according to the different age groups. In the first age group, $62.5 \mathrm{mg} / \mathrm{day}$ was the mean of the daily doses of furosemide, and it decreased in the second group till it reached $40.49 \mathrm{mg} /$ day. However, it increased in the third group to reached 74.29 $\mathrm{mg}$ /day, while in the fourth group it decreased again to reached to $65.45 \mathrm{mg} / \mathrm{day}$. There was no significant difference in the means between the age groups $(P$ value $=$ $0.19)$. 


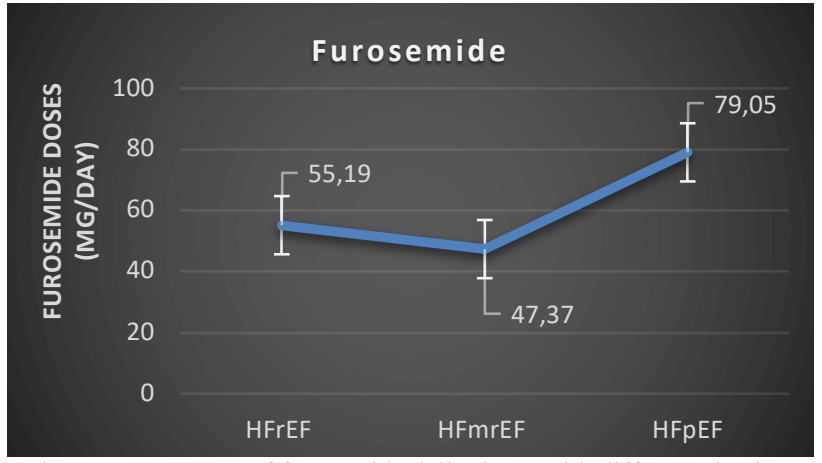

Fig. 17. Mean \pm SE of furosemide daily doses with different ejection fraction groups.

(HFrEF) heart failure with reduced ejection fraction $(<40 \%)$, (HFmrEF) heart failure with mid-range ejection fraction (40-49\%), (HFpEF) heart failure with preserved ejection fraction $(>49 \%)$.

Fig. 17 illustrates the change in the mean of daily doses of furosemide prescribed to heart patients according to different ejection fraction groups. The mean of daily doses of furosemide for HFrEF was $55.19 \mathrm{mg} /$ day, and it decreased slightly to $47.37 \mathrm{mg} /$ day in the second group. However, it increased dramatically to reach $79.05 \mathrm{mg} /$ day in HFpEF group. This fluctuation shows no significant $(P$ value $=0.064)$.

\section{Hydrochlorothiazide}

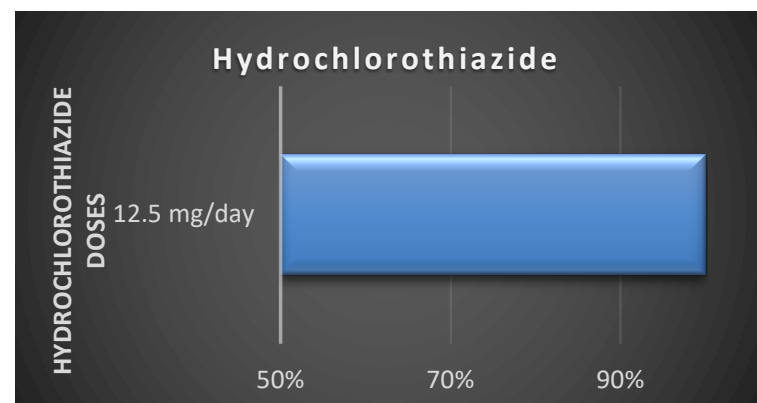

Fig. 18. Percentage of hydrochlorothiazide daily doses prescribed for heart failure patients in SQUH.

Fig. 18 shows that dose of hydrochlorothiazide was prescribed for 8 patients as $12.5 \mathrm{mg} /$ day.

\section{J. Spironolactone}

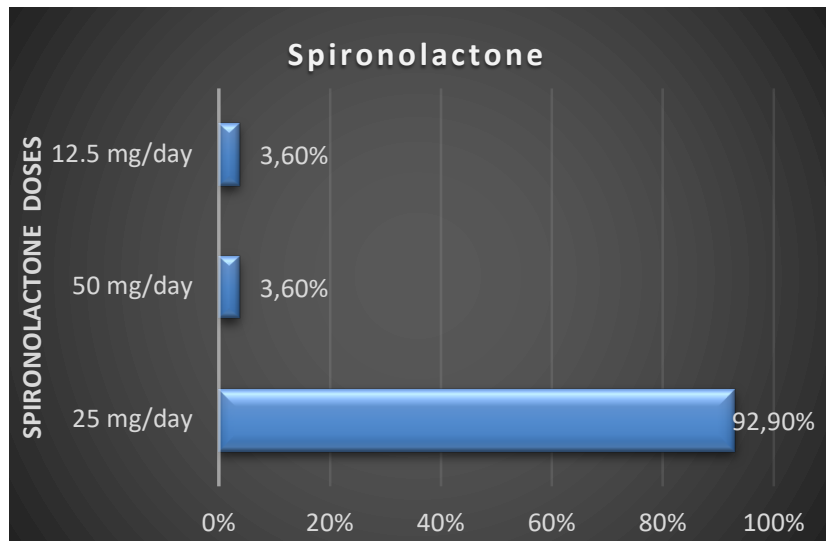

Fig. 19. Percentage of spironolactone daily doses prescribed for heart failure patients in SQUH

Fig. 19 shows the daily doses of spironolactone given to heart failure patients. There were 3 doses of spironolactone $12.5,25$ and $50 \mathrm{mg} /$ day. $92.90 \%$ (52 patients out of 56) were on $25 \mathrm{mg}$ /day of spironolactone, $3.60 \%$ (2 patients out of
56) were on $50 \mathrm{mg} / \mathrm{day}$, and $3.60 \%$ (2 patients out of 56) were on $12.5 \mathrm{mg} /$ day.

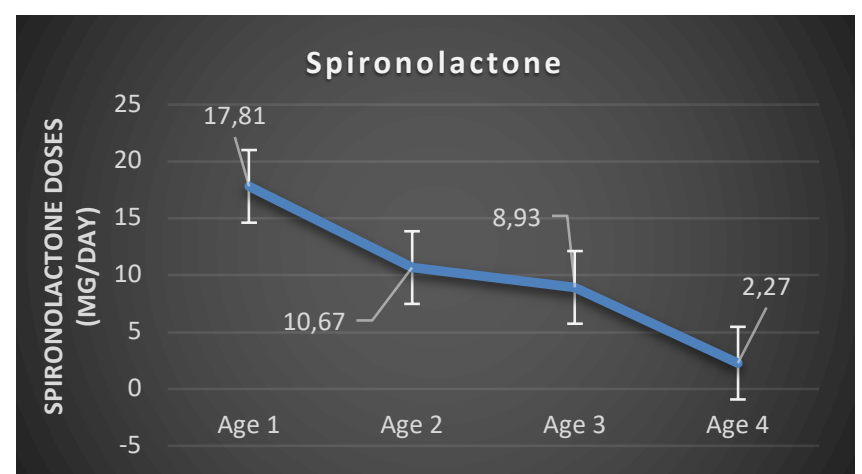

Fig. 20. Mean \pm SE of spironolactone daily doses with different age groups. (Age 1) $<60$ years, (Age 2) 60-70 years, (Age 3 ) 70-80 years, (Age 4) $>80$ years. (NYHA) New York Heart Association.

Fig. 20 illustrates the change in the mean of daily doses of spironolactone prescribed to heart failure patients according to different age groups. The mean of the daily doses for the first age group was $17.81 \mathrm{mg} / \mathrm{day}$, and it decreased in the second age group to reach $10.67 \mathrm{mg} / \mathrm{day}$, and it continued to decrease in the third and fourth age groups to reach 8.93 $\mathrm{mg}$ /day and $2.27 \mathrm{mg} /$ day respectively. There was a significant difference in the utilization of spironolactone in the different age groups $(P$ value $=0.001)$.

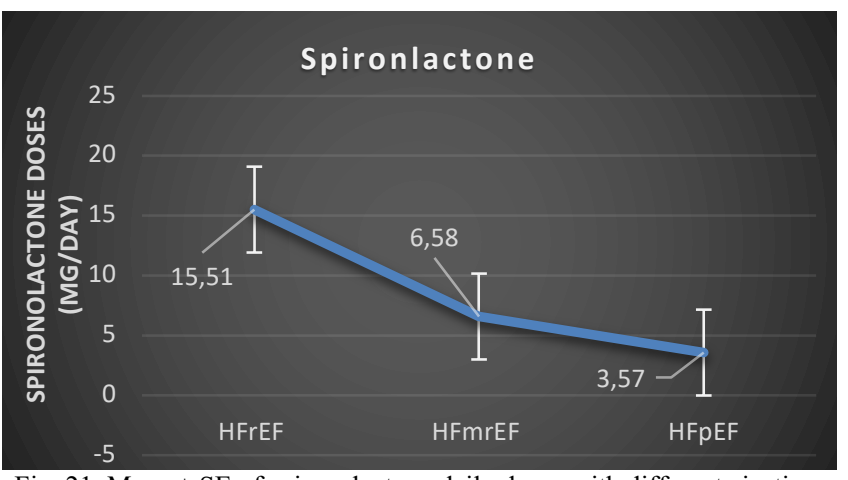

Fig. 21. Mean \pm SE of spironolactone daily doses with different ejection fraction groups

(HFrEF) heart failure with reduced ejection fraction $(<40 \%)$, (HFmrEF) heart failure with mid-range ejection fraction $(40-49 \%)$, (HFpEF) heart failure with preserved ejection fraction $(>49 \%)$

Fig. 21 shows the change in the mean of daily doses of spironolactone prescribed to heart failure patients according to different ejection fraction groups. The mean daily doses of HFrEF were $15.51 \mathrm{mg} /$ day and it continued to decrease in HFmrEF and HFpEF to reach $6.58 \mathrm{mg} /$ day and $3.57 \mathrm{mg} /$ day respectively. There was a significant difference in the utilization of spironolactone in different ejection fraction groups $(P$ value $=0.001)$.

\section{DISCUSSION}

This research discusses the utilization of beta-blockers and diuretics used in treating heart failure patients in Sultan Qaboos University Hospital (SQUH). The study included 120 patients diagnosed with heart failure and have been treated in SQUH. All the patients were Omanis as SQUH is considered as tertiary and governmental hospital, and most of the patients who get treated in this hospital are Omanis. The ages of 120 patients were collected and showed that 
they range from 25 to 92 years with mean \pm standard deviation equals to $64 \pm 13$, this result follow our expectation as heart failure is well known as age-related disease (Rich, 1997). From 120 patients, we found that most of them have heart failure reduced ejection fraction and similar finding has been mentioned in previous studies (Agarwal et al., 2001). We noticed that smokers and alcoholic patients were 23 out of 120 which is reasonable because of religious reasons. We were able to collect 12 NYHA classification for 12 patients and we found that most of them were in class II and III, and similar finding also has been seen in a study was conducted in Henan University Huaihe Hospital (china) (Xiang S et al, 2016). That study found that out of 66 patients 5 were having NYHA class II and 46 having NYHA class III in both control and cases patients collectively. It seems that hypertension and diabetes mellitus type 2 have the highest prevalence among heart failure patients with percentage equal to $62.5 \%$ and $40.8 \%$ respectively, and we have no doubt about this as they are prevalent in Omani society.

\section{A. Beta-blockers}

Many RCTs mentioned that beta-blockers have shown their effectiveness in reducing mortality by $29 \%$ and cardiovascular hospitalization (A National Clinical Guideline, 2016). By studying the percentage of utilization of beta-blockers medications among heart failure patients in SQUH, we found that $81.6 \%$ of our patients were on betablockers. $62.2 \%$ were on carvedilol and $37.8 \%$ were on bisoprolol. Carvedilol and bisoprolol are the drugs of choice of beta-blockers for treating heart failure patients so that why SQUH using those two drugs. We found that, the utilization of beta-blockers medication decreased significantly with increase in the age of the heart failure patients, however some studies showed that beta-blockers are effective in treating the older heart failure patients which disagree with our finding but the comorbidities that our patients have and the ability of our patients to tolerate the drugs may lead to decrease the utilization of beta-blockers drugs. Meanwhile there was insignificant decrease in the utilization of beta-blockers with increase in ejection fraction and that was noticed in the previous clinical practices and trails, the exact reason is unknown it could reflect the treatment of cardiovascular co-morbidities or failure in distinguish between HFrEF, HEmEF and HFpEF (Liu et al, 2014).

\section{B. Bisoprolol}

The results showed that $37.8 \%$ of our patients were on bisoprolol, and most of them were on 5 and $2.5 \mathrm{mg} /$ day with percentage equal to $39.5 \%$ and $36.80 \%$ respectively, however $13.2 \%$ were on $10 \mathrm{mg}$ /day and $10.5 \%$ were on 7.5 . These results showed that SQUH pharmacy follows the 2016 European Society of Cardiology Guidelines for the diagnosis and treatment of acute and chronic heart failure which stated that the starting dose is 1.25 and the target dose is $10 \mathrm{mg}$, and most of our patient were on 2.5 and $5 \mathrm{mg} /$ day possibly because they could not tolerate the higher doses or because their heart failure worsen with higher doses (BNF, 2015). The mean daily doses of bisoprolol were not affected neither by change the age nor by ejection fraction with $p$ value equal to 0.41 and 0.565 respectively.

\section{Carvedilol}

$62.2 \%$ of our patients were on carvedilol and most of them were on 25 and $50 \mathrm{mg}$ /day with percentage equal to $47.7 \%$ and $41 \%$ respectively, $21.3 \%$ of them were on 12.5 and $6.25 \mathrm{mg} /$ day. Those doses are similar to what has been mentioned on 2016 European Society of Cardiology Guidelines for the diagnosis and treatment of acute and chronic heart failure. The target dose is $3.125 \mathrm{mg} /$ day to 25 $\mathrm{mg} /$ day and it can reach to $50 \mathrm{gm} /$ day for patients who weight above $85 \mathrm{~kg}$. The mean daily doses of carvedilol decreased significantly with change in the age group ( $p$ value $=0.006$ ), and this could be because of the contradictions and wide effects of carvedilol which is nonselective beta-blocker with alpha receptor blocker characteristic (Westfall \& Westfall, 2011). Changes in the ejection fraction does not effects the mean daily doses of carvedilol and there is no clear reason behind that.

\section{Diuretics}

A meta-analysis study was done on diuretics therapy and showed a reduction in mortality and improve exercise ability by $75 \%$ and $63 \%$ respectively (A National Clinical Guideline,2016). The doses of diuretics should be individualized, so the dose of diuretics should able to eliminate the symptoms of fluid retention and not causing dehydration or hypotension to the patients (A National Clinical Guideline,2016). Our study revealed that 114 out of 120 patients were on diuretic drugs. $93.9 \%$ were on furosemide, $49.1 \%$ were on spironolactone, and $7 \%$ were on hydrochlorothiazide. Utilization of diuretics were not affected significantly neither by change the age nor by ejection fraction $(p$ value $=0.917)$.

\section{E. Furosemide}

Furosemide seem to be preferred over any other types of diuretics where $93.9 \%$ of our patients were on it, and that because their potency and the ability to maintain their diuretic effects even at lower estimated renal function (Dickstein et al, 2008). Nine doses of furosemide were taken by our patients, range from 20 to $240 \mathrm{mg} /$ day. $59.9 \%$ of our patients were on $40 \mathrm{mg}$ /day while $16.8 \%$ were on 80 $\mathrm{mg}$ /day, the remaining patients were distributed among the different doses. It seems that the utilization of furosemide in SQUH also follow the 2016 European Society of Cardiology Guidelines for the diagnosis and treatment of acute and chronic heart failure which stated that the usual daily doses ranging from $40-240 \mathrm{mg} /$ day. Utilization of furosemide shows no significant decrease or increase in the mean daily doses with change in the age and ejection fraction.

\section{F. Hydrochlorothiazide}

Hydrochlorothiazide was prescribed for only 8 patients in fix dose combination and all of them were on $12.5 \mathrm{mg} /$ day, and that is because of Hydrochlorothiazide electrolytes imbalance effects (Rang et al., 2011). However, 2016 European Society of Cardiology Guidelines for the diagnosis and treatment of acute and chronic heart failure showed that the daily doses of hydrochlorothiazide ranging from 12.5 to $100 \mathrm{mg}$. we could not compare the mean of doses with age and ejection fraction of the patients as it's fix dose combination. 


\section{G. Spironolactone}

Spironolactone is the second most prescribed diuretics among our patients, and that could be because of their aldosterone antagonist activities (Rang et al., 2011). Our results showed that $92.90 \%$ of our patients were on 25 $\mathrm{mg}$ /day of spironolactone and $7.2 \%$ on 12.5 and $50 \mathrm{mg} /$ day. However, the 2016 European Society of Cardiology Guidelines for the diagnosis and treatment of acute and chronic heart failure stated that the daily doses are from 25 $\mathrm{mg}$ /day till $50 \mathrm{mg} /$ day. $3.6 \%$ of our patients were on 12.5 $\mathrm{mg} /$ day and that could be because they ca not tolerate the 25 mg. A significant decrease in the utilization of spironolactone with the increase in the age was noticed ( $p$ value $=0.001)$ and could be because that those patients are at high risk of electrolytes imbalance or because of the comorbidities that patients have, also with increase in the ejection fraction there was a significant decrease $(p$ value $=$ 0.001 ) as most of HFpEF do not suffer from fluid retention symptoms (A National Clinical Guideline, 2016).

\section{CONCLUSION}

Carvedilol and furosemide were the most used drugs in treating heart failure patients in SQUH. All the mean daily doses of the drugs mentioned in this study followed the newer 2016 European Society of Cardiology Guidelines for the diagnosis and treatment of acute and chronic heart failure. Carvedilol and spironolactone utilization seem to be affected by increase patients age, however spironolactone utilization only seem to be affected by increase the ejection fraction. The least diuretics prescribed is hydrochlorothiazide and the least beta-blocker prescribed is bisoprolol.

\section{ABBREVIATIONS}

\begin{tabular}{cc}
\hline \hline The abbreviation & The meaning \\
\hline SQUH & Sultan Qaboos university hospital \\
\hline BNP & Brain Natriuretic Peptide \\
\hline eGFR & Estimated Glomerular Filtration Rate \\
\hline EF & Ejection Fraction \\
\hline HF & Heart Failure \\
\hline HFmrEF & Heart Failure with Mid-Range Ejection Fraction \\
\hline HFpEF & Heart Failure with Preserved Ejection Fraction \\
\hline HFrEF & Heart Failure with Reduced Ejection Fraction \\
\hline STEMI & ST-Elevated Myocardial Infarction \\
\hline NSTEMI & Non-ST-Elevated Myocardial Infarction \\
\hline SPSS & Statistical Package for the Social Sciences program \\
\hline NYHA & New York Heart Association \\
\hline SD & Standard deviation \\
\hline SE & Standard error \\
\hline ESC & 2016 European Society of Cardiology \\
\hline \hline
\end{tabular}

\section{REFERENCES}

[1] 2016 ESC Guidelines for the diagnosis and treatment of acute and chronic heart failure, European Heart Journal Advance Access published June 8, 2016

[2] A National Clinical Guideline. SIGN 147; Management of chronic heart failure; 2016; 18-23.

[3] Agarwal AK, Venugopalan P, de Bono D. Prevalence and etiology of heart failure in an Arab population. Eur J Heart Failure 2001; 3: 301305 .
[4] Al-Shamiri MQ. Heart Failure in the Middle East. Current Cardiology Reviews, 2013, 9, 174-178.

[5] British National Formular; 2015

[6] Bruns LA, Carter CE (2002) Should B-blockers be used for the treatment of pediatric patients with chronic heart failure? Pediatr Drugs 4:771-778.

[7] Dickstein K, Cohen-Solal A, Filippatos G. ESC Guidelines for the diagnosis and treatment of acute and chronic heart failure. Eur Heart $\mathbf{J}$ 2008; 29: 2388-442.

[8] Doba N, Tomiyama H, Nakayama T. Drugs, Heart Failure and Quality of Life. Drugs \& Aging 1999 Mar; 14 (3): 153-163.

[9] He, Y., Yang, X., Zhao, X., Cheng, X., Xu, H., Qian, Y. and Li, X. $\beta$ Blockers in Heart Failure: Benefits of $\beta$-Blockers according to varying male proportions of study patients. Clin Cardiol, 2012; 35(8):505-511

[10] Hunt SA, Abraham WT, Chin MH, Feldman AM, Francis GS, Ganiats TG, Jessup M, Konstam MA, Mancini DM, Michl K, Oates JA, Rahko PS, Silver MA, Stevenson LW, Yancy CW, Antman EM, Smith SC, Jr, Adams CD, Anderson JL, Faxon DP, Fuster V, Halperin JL, Hiratzka LF, Jacobs AK, Nishimura R, Ornato JP, Page RL, Riegel B. ACC/AHA 2005 Guideline Update for the Diagnosis and Management of Chronic Heart Failure in the Adult: a report of the American College of Cardiology/ American Heart Association Task Force on Practice Guidelines. Circula- tion 2005; 112:e154-235.

[11] Laer S, Mir TS, Behn F et al (2002) Carvedilol therapy in pediatric patients with congestive heart failure: a study investigating clinical and pharmacokinetic parameters. Am Heart J 143(5):916-922.

[12] Levy E. \& LevyP. Pharmacoeconomic Considerations in Assessing and Selecting Congestive Heart Failure Therapies. Pharmacoeconomics 2002; 20 (14): 963-977.

[13] Liu F, Chen Y, Feng X, et al. Effects of Beta-Blockers on Heart Failure with Preserved Ejection Fraction: A Meta-Analysis, 2014; PLoS ONE 9(3): e90555.

[14] McMurray JJV; Adamopoulos S; Anker SD et al. ESC Guidelines for the diagnosis and treatment of acute and chronic heart failure 2012, The Task Force for the Diagnosis and Treatment of Acute and Chronic Heart Failure 2012 of the European Society of Cardi- ology. European Heart Journal 2012; 33: 1787-1847.

[15] Mosterd A, Hoes AW. Clinical epidemiology of heart failure. Heart 2007; 93: 1137-1146.

[16] Nicholson C.Chronic heart failure: pathophysiology, diagnosis and treatment. Nursing Older People.2014; 26, 7, 29-38.

[17] Packer M, Bristow MR, Cohn JN, Colucci WS, Fowler MB, Gilbert EM, Shusterman NH. The effect of carvedilol on morbidity and mortality in patients with chronic heart fail- ure. N Engl J Med 1996; 334:1349-1355.

[18] Rang HP, Dale MM, Ritter JM. Pharmacology, 7th Edition. Churchill Livingstone, Edinburgh 2011.

[19] Rich MW. Epidemiology, pathophysiology, and etiology of congestive heart failure in older adults. J Am Geriatr Soc 1997; 45:968-974.

[20] Shihmanter R, Nulman I, Goland S, et al. Pharmacogenetics: Variation in the CYP2D6 genotype is not associated with carvedilol dose changes in patients with heart failure. Journal of Clinical Pharmacy and Therapeutics, 2014; 39;432-438.

[21] Trobec C, Grabnar1 I, Kos K, et al. Bisoprolol pharmacokinetics and body composition in patients with chronic heart failure: a longitudinal study. Eur J Clin Pharmacol, 2016; 72:813-822.

[22] Westfall TC, Westfall DP (2011) Adrenergic agonists and antagonists. In: Brunton LL, Chabner BA, Knollmann BC (eds) Goodman and Gilman's the phar- macological basis of therapeutics, 12th edn. McGraw- Hill, New York.

[23] Xiang S, Zhang N, Yang Z, et al. Achievement of a target dose of bisoprolol may not be a preferred option for attenuating pressure overload-induced cardiac hypertrophy and fibrosis. Experimental And Therapeutic Medicine, 2016; 12: 2027-2038. 